\title{
The Influence of Environmental Regulation on the Innovation Performance of Manufacturing Listed Companies Based on the Difference of Government Support
}

\author{
Jiang Zhangsheng ${ }^{12}$ Zhou Cuiying $^{1}$ \\ ${ }^{1}$ Zhejiang Gongshang University School of Business Administration, \\ ${ }^{2}$ Zhejiang Gongshang University Zhejiang merchants Research Center, Zhejiang Hangzhou \\ 310018
}

Keywords: government support; environmental regulation; tax preferences; innovation performance

\begin{abstract}
The difference in the intensity of local government support determines the impact of environmental regulation on the innovation performance of enterprises. Using the micro-level data of 104 listed companies in the 2011-2015 manufacturing industry, this paper examines how the difference between the intensity of government direct subsidy and tax incentives affects the relationship between environmental regulation and innovation performance. The results show that: The environmental regulation has a significant positive impact on the innovation performance of manufacturing companies when the government tax preferential intensity is high.
\end{abstract}

\section{政府支持差异性下环境规制对制造业上市公司创新绩效的影响}

\author{
蒋樟生 12 周粹芗 1 \\ 1 浙江工商大学工商管理学院, \\ 2 浙江工商大学浙商研究中心, 浙江 杭州 310018) \\ 975762260@qq.com
}

关键词：政府支持；环境规制；税收优惠；创新绩效

摘要. 政府支持的差异性决定了环境规制对企业创新绩效的影响存在不同。基于环境规制对 企业创新绩效的不同作用理论分析, 利用2011-2015年制造业104家上市公司的微观层面数据, 考察政府直接资金补贴与税收优惠的强度差异性下环境规制如何影响制造业上市公司的创新 绩效。研究发现: 当政府税收优惠强度较高时, 环境规制对制造业上市公司创新绩效产生显 著正向影响。

\section{1 引言}

自改革开放以来, 中国把重心放在了经济发展上, 忽视了环境方面污染严重的问题, 这 不利于中国的可持续发展目标的实现。面对日益恶化的环境质量，国家开始不断重视环境方 面的问题，提出“绿色发展”的核心理念以改变现有的粗放型经济发展方式，强调走绿色生产 和绿色治理相结合的生态文明之路, 试图实现环境与经济的“双赢”效果。截至“十二五”末期, 国家累计发布环保标准 1941 项。环境规制政策的实施必然会影响经济的发展，但其与企业创 新之间的关系仍然具有不确定性 ${ }^{[1]}$ 。Mohr 和贾瑞跃等是“波特假说”的支持者，环境规制会倒 
逼企业进行创新 ${ }^{[2][3]}$; 而 Chintrakarn 和王玉振等则是新古典经济学派的支持者, 环境规制会

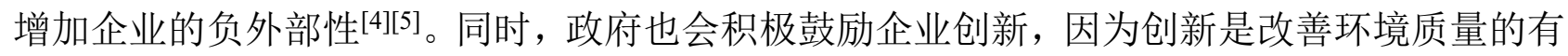
效途径。Guellec 和张同斌等是凯恩斯经济学理论和熊彼特技术创新理论支持者, 认为政府支 持可以促进企业创新 ${ }^{[6][7]}$; Bloom 和余泳泽等则支持信息不对称理论、代理理论以及挤出效应 理论，认为政府支持不利于企业创新 ${ }^{[8][9]}$ 。

鉴于环境规制政策的实施不可避免会给企业带来治理成本，削弱企业利润，挤出企业研 发投入。经济新常态下的创新驱动又要求企业寻求在环境规制强度提高的情形下能够降低环 境治理成本、促进创新的方法，此时若能得到政府支持，则能够较好解决环境规制对企业创 新的制约问题。因此，在对现有相关文献梳理的基础上，基于“波特假说”、新古典经济学以 及制度理论等理论, 利用我国制造业上市公司 2011-2015 年微观层面的面板数据, 探讨环境 规制与政府支持是否都能够促进企业创新绩效的提高, 政府支持强度增大是否能够促进环境 规制对于企业创新绩效的影响?

\section{2. 理论分析}

\section{1 环境规制对企业创新绩效的不同作用机制}

首先, 从新古典经济学角度来看, 在理性决策的假设下, 环境规制将不可避免地增加企 业制造成本，从而降低企业创新效率[10]。同时，环境治理也可能挤出生产投资从而降低企业 创新绩效 ${ }^{[11]}$ 。Mateescu 等认为一旦实施环境规制措施, 企业就要承担造成环境损失的赔偿责 任, 并且还要在人力资源、设备和资金方面进行额外投资, 以使其制造过程完全或至少部分 地适应环境法规政策的要求，这样会使企业创新投入减少，创新绩效将会下降[12]。

其次, 有大量的学者认为有效的环境规制将鼓励企业积极进行创新。国内外学者在“波特 假说” 的逻辑框架下进行了大量的理论与实证研究, 认为环境规制可以降低企业的创新成本, 增强企业竞争力，从而使企业建立“先发优势”[2][3]。同时，沈能和李娜等发现短期内环境规制 并不一定总是能够补偿成本带来的负外部性，但是长期内是有效的 ${ }^{[13][14]}$ 。

\section{2 政府支持对企业创新绩效的不同作用机制}

技术创新理论和资源基础理论认为政府支持可以通过直接的创新资源补充机制影响企业 创新行为。政府通过采取相应的研发补贴、税收优惠与政策激励等手段去降低创新的不确定 性和成本, 进而促进企业的研发投入与创新 ${ }^{[15]}$ 。Kleer 和郭晓丹等学者从信号理论的角度出发, 政府支持会向外部释放利好信号, 帮助企业贴上被政府认可的标签, 这样不仅获得了政府支 持, 还更有机会获得其他渠道的支持，进而帮助企业获取所需创新资源提升创新绩效 ${ }^{[16][17] 。}$

信息不对称与代理理论认为，政府与企业之间存在信息不对称和委托代理问题，导致资 源无法达到最优配置 ${ }^{[18]}$ 。Murphy 等认为政府在给企业分配补贴过程中容易滋生寻租行为, 破 坏市场正常的竞争秩序，出现企业套取各种政府补贴并将资金挪作他用，最终会抑制企业的 技术创新 ${ }^{[19]}$ 。范允奇等运用 2000-2011 年中国高技术产业的省际面板数据进行分析, 认为政 府研发投入与技术创新效率之间关系不显著，政府研发投入对技术创新效率的空间外溢效应 表现为发达地区对不发达地区的负向空间外溢 ${ }^{[20]}$ 。目前, 对于政府支持与创新绩效之间的关 系仍未达成共识, 需要进一步深入探讨。

\section{3 环境规制、政府支持与企业创新绩效之间的作用机制}

在经济转型时期的中国, 环境规制与政府支持都会显著影响企业创新行为及其绩效。而 且, 目前我国的市场机制还不够完善, 政府承担了部分资源管理的职能, 在此背景下, 政府 提供创新支持可以有效降低环境规制给企业创新带来的成本和不确定性。当政府提供资金补 贴或者一些税收优惠时, 企业会获得某种利好信号, 从而增强企业创新动力 ${ }^{[16]}$ 。同时, 为了 获得更多相关支持, 企业会遵守各种规章制度, 配合政府工作, 其中包括目前国家最重视的 
环境质量改善工作，改善生产工艺和流程以实现环境与经济效益的双赢效果。

孙伟等利用 35 个行业的面板数据, 研究发现环境规制对于企业创新呈现出“U”型作用, 若在环境规制的同时增加政府投入，可以显著的促进企业创新 ${ }^{[21]}$ 。但是，从微观企业角度将 环境规制与政府支持结合一起研究其创新效应的文献还比较缺乏，因此研究政府支持对于环 境规制创新效应的影响具有重要理论意义和实践价值。

\section{3. 计量模型设计与变量描述统计}

\section{1 模型设定}

基于上述文献研究的理论基础和经验假设分析，结合波特假说和凯恩斯经济学理论建立 如下模型，以我国制造业上公司为研究对象分析政府支持、环境规制与企业创新绩效三者之 间的作用关系。

$$
\begin{gathered}
\text { inno }_{i t}=\alpha_{1}+\beta_{11} e r_{i t}+\beta_{12} g d s_{i t}+\beta_{13} g t p_{i t}+\varepsilon_{1 i t} \\
\text { inno }_{i t}=\alpha_{2}+\beta_{21} e r_{i t}+\beta_{22} g d s_{i t}+\beta_{23} g t p_{i t}+\text { control }+\varepsilon_{2 i t}
\end{gathered}
$$

式中 $i$ 表示企业, $t$ 表示时间, inno 表示企业创新绩效, $e r$ 表示环境规制强度, $g d s$ 是研 发前政府提供的直接资金补贴强度, gtp 是政府对企业创新所提供的税收优惠强度, control 是 控制变量, $\varepsilon$ 是随机干扰项。

同时，考虑到环境规制对企业技术创新的影响能够以非线性的形式表现出来 ${ }^{21]}$ 。因此， 在模型 3 引入环境规制的平方项:

$$
\text { inno }_{i t}=\alpha_{3}+\beta_{31} e r_{i t}+\beta_{32} e r^{2}{ }_{i t}+\beta_{33} g d s_{i t}+\beta_{34} g t p_{i t}+\text { control }+\varepsilon_{3 i t}
$$

最后，为了检验政府支持强度差异是否能够影响环境规制强度与企业创新绩效之间的关 系。因此, 在模型 4 和 5 中引入乘积项对应的计量模型如下:

$$
\begin{gathered}
\text { inno }_{i t}=\alpha_{4}+\beta_{41} e r_{i t}+\beta_{42} e r_{i t}^{2}+\beta_{43} g d s_{i t}+\beta_{44} g t p_{i t}+\beta_{45} e r_{i t} \times g d s_{i t}+\text { control }+\varepsilon_{4 i t} \\
\text { inno }_{i t}=\alpha_{5}+\beta_{51} e r_{i t}+\beta_{52} e r^{2}{ }_{i t}+\beta_{53} g d s_{i t}+\beta_{54} g t p_{i t}+\beta_{55} e r_{i t} \times g t p_{i t}+\text { control }+\varepsilon_{5 i t}
\end{gathered}
$$

若政府支持强度能够促进环境规制与创新绩效之间的关系，则模型(4)和(5)中乘积项的回 归系数大于零，表明当政府支持强度增加时，环境规制强度能够正向促进企业创新绩效。

\section{2 变量说明}

企业创新绩效 (inno): 反映企业技术创新产出水平的指标主要包括申请专利数、新产品 销售收入和授权专利数。由于新产品的统计口径和定义并不清晰, 不利于准确获得企业新产 品销售收入; 同时，一项专利从申请到授权大约需 1-2 年的时间，其值存在一定的滞后性。 且考虑到研究样本区间为 2011-2015 年以及数据的可获得性, 采用企业当年专利申请数量来 衡量。

环境规制强度(er): 现有研究主要有以下四种方式来测度微观企业层面的环境规制强度, 一是用企业单位产出的污染治理费用来衡量; 二是用企业接受政府环保检查的时间以及受到 环保部门的罚款来表示; 三是利用某种污染物的排放密度或者不同污染物的排放密度作为代 理变量; 四是使用李克特量表构造企业环境规制的指标对企业管理者进行问卷调查。考虑到 微观企业数据的可获得性和制造企业的特殊性，采用企业排污费来衡量。

政府直接资金补贴强度 $(g d s)$ ：企业进行研发活动前，政府对企业创新的支持强度，通过 政府直接资金补贴来衡量。

政府税收优惠强度 $(g t p)$ : 政府为了鼓励企业改善生产工艺和流程, 使其改善环境质量, 会对企业创新进行一定的税收优惠，以企业收到的税费返还来衡量。

企业规模( size ): 政府科技资助激励企业研发投入的效果会受到企业规模的影响，采用企 业总资产来衡量。

企业盈利能力 (pro): 企业盈利能力直接决定了企业可用资金, 会对企业创新产生一定的 
影响，采用企业利润总额占营业收入的比值来衡量。

企业研发费用 $(r d)$ : 企业研发费用的投入会很大程度影响企业研发产出, 采用企业研发 费用占营业收入的比值来衡量。

企业人力资本 $(h c)$ ：企业人力资本是影响企业技术创新能力的重要因素。企业的技术创 新需要高水平的研发人员, 企业的技术创新是核心技术人员创造性成果的体现, 企业工资水 平越高表明企业人力资本越高。因此，采用企业应发工资作为替代指标来衡量。

\section{3 数据来源与描述性统计}

样本选择过程和数据来源如下: 首先, 以沪深证券市场中证监会行业分类中的制造业上 市公司为样本, 在对 2011-2015 年全部制造业上市公司的财务报表进行详细分析的基础上, 剔除了 ST 和数据严重缺失的数据, 以及明显存在异常的数据。因此。最终进入分析的是 104 家制造业上市公司数据。借鉴已有文献的做法，企业创新绩效采用企业当年专利申请数量来 衡量, 这些数据都通过从 SooPat 专利检索网手工搜索获得。企业排污费从各省的环境保护局 网站手动搜集整理获得。企业研发费用、资产总值、政府补贴费用, 收到的税收返还费用以 及盈利数据等数据均从同花顺数据库获取, 对于个别缺失的数据, 从企业年报手工搜集整理 获得。在此, 对各个变量指标的数据特征, 包括最小值、最大值、均值和标准差, 进行了详 细的描述，具体结果如表 1 所示。

表 1 变量描述统计

\begin{tabular}{c|c|c|c|c}
\hline 指标 & 最小值 & 最大值 & 均值 & 标准差 \\
\hline inno & 0 & 369.000 & 12.681 & 41.294 \\
\hline$e r$ & 0 & 7.734 & 0.379 & 0.892 \\
\hline$e r^{2}$ & 0 & 59.812 & 0.938 & 14.577 \\
\hline$g d s$ & 0.012 & 200.176 & 6.366 & 6.600 \\
\hline$g t p$ & 0 & 38.200 & 3.478 & 16.830 \\
\hline$s i z e$ & 0.410 & 102.988 & 11.588 & 0.131 \\
\hline$p r o$ & -0.715 & 0.707 & 0.059 & 1.660 \\
\hline$r d$ & 0.004 & 9.440 & 2.359 & 24.000 \\
\hline$h c$ & 0.053 & 238.705 & 9.740 & 55.751 \\
\hline$e r \times g d s$ & 0 & 1163.506 & 6.983 & 14.760 \\
\hline
\end{tabular}

\section{4. 实证过程及结果分析}

基于我国制造业上市公司全部样本进行面板数据模型的选择及回归结果见表 2 ，圥余性 检验和豪斯曼检验结果显示模型(1)到模型(5)都支持截面固定效应模型。

表 2 环境规制、政府支持对企业创新绩效的影响

\begin{tabular}{c|c|c|c|c|c}
\hline 变量 & 模型(1) & 模型(2) & 模型(3) & 模型(4) & 模型(5) \\
\hline$e r$ & $-0.007^{* * *}$ & $-0.132^{* * *}$ & -0.008 & -0.022 & 0.024 \\
\hline$e r^{2}$ & & & $-0.088^{*}$ & $-0.091^{*}$ & $-0.083^{*}$ \\
\hline$g d s$ & $0.040^{* *}$ & $0.0410^{* *}$ & $0.0430^{* * *}$ & 0.0200 & $0.0420^{* * *}$ \\
\hline$g t p$ & $-0.0758^{* * *}$ & -0.033 & -0.030 & -0.0340 & $-0.2360^{* * *}$ \\
\hline size & & $-0.265^{* * *}$ & $-0.261^{* * *}$ & $-0.239^{* * *}$ & $-0.201^{* * *}$ \\
\hline$p r o$ & & 0.029 & 0.031 & 0.031 & $0.089^{* * *}$ \\
\hline$r d$ & & 0.037 & 0.039 & 0.046 & 0.033 \\
\hline$h c$ & & $-0.120^{* * *}$ & $-0.153^{* * *}$ & $-0.122^{* * *}$ & -0.024 \\
\hline$e r \times g d s$ & & & & 0.0299 & \\
\hline$e r \times g t p$ & & & & & $0.1675^{* * *}$ \\
\hline
\end{tabular}


表 2 续 环境规制、政府支持对企业创新绩效的影响

\begin{tabular}{|c|c|c|c|c|c|}
\hline$R^{2}$ & 0.948 & 0.954 & 0.955 & 0.955 & 0.963 \\
\hline $\mathrm{F}$ 统计量 & $\begin{array}{c}71.252 \\
(0.000)\end{array}$ & $\begin{array}{c}77.092 \\
(0.000)\end{array}$ & $\begin{array}{c}76.939 \\
(0.000)\end{array}$ & $\begin{array}{c}77.002 \\
(0.000) \\
\end{array}$ & $\begin{array}{c}89.366 \\
(0.000)\end{array}$ \\
\hline DW 统计量 & 1.725 & 1.701 & 1.770 & 1.594 & 1.635 \\
\hline 圥余性检验 & $\begin{array}{c}49.834 \\
(0.0000) \\
\end{array}$ & $\begin{array}{c}42.933 \\
(0.0000) \\
\end{array}$ & $\begin{array}{c}40.536 \\
(0.0000) \\
\end{array}$ & $\begin{array}{c}40.166 \\
(0.0000)\end{array}$ & $\begin{array}{c}47.371 \\
(0.0000) \\
\end{array}$ \\
\hline 豪斯曼检验 & $\begin{array}{l}107.666 \\
(0.0000)\end{array}$ & $\begin{array}{l}200.461 \\
(0.0000)\end{array}$ & $\begin{array}{l}340.956 \\
(0.0000)\end{array}$ & $\begin{array}{c}356.111 \\
(0.0000)\end{array}$ & $\begin{array}{c}399.399 \\
(0.0000) \\
\end{array}$ \\
\hline 模型选择 & 固定效应 & 固定效应 & 固定效应 & 固定效应 & 固定效应 \\
\hline
\end{tabular}

注: ${ }^{* * *}$ 表示在 $1 \%$ 的显著性水平下通过了检验, ${ }^{* *}$ 表示在 $5 \%$ 的显著性水平下通过了检验，“表 示在 $10 \%$ 的显著性水平下通过了检验。

从表 2 模型(1)、(2)的回归系数来看, 无论是否控制其他变量, 环境规制强度与企业创新 绩效之间的关系都是显著负向的，说明对我国制造业上市公司而言，环境规制强度越大，越 容易给企业增加环境控制成本，越不利于企业进行创新。从表 2 模型(3)、(4)、(5)的回归系数 来看, 环境规制强度平方项的回归系数显著为负，说明环境规制与企业创新绩效之间呈现倒 “U”型关系，说明适当的环境规制可以倒逼企业进行创新，但是环境规制强度如果过大，企业 创新带来的效益将无法弥补过高的环境控制成本，会阻碍企业创新。表 2 模型(1)到模型(5) 显示政府直接资金补贴强度对环境规制强度的回归系数为正，且基本都是显著的，说明政府 的直接资金补贴可以激励企业创新。然而，政府税费优惠强度与企业创新绩效之间的关系呈 现负向关系, 说明政府所提供的税收优惠政策对于我国制造业上市公司的创新激励作用不但 没有增强, 反而减弱了, 但作用不够显著, 这说明制造企业在创新前需要强有力的资金支持, 税收优惠对其创新行为的激励作用较为有限。

表2模型(4)的交互项回归系数为正，说明当政府直接资金补贴强度较高时，环境规制可 以正向促进企业创新绩效，但是其作用并不明显。这可能有两个方面的原因，第一，环境规 制对于企业所造成负外部性太强，政府直接的资金补贴无法弥补负外部性; 第二，仅仅依靠 政府直接资金补贴是不够的, 企业本身必须具有相应的创新意识与创新能力。表2模型(5)中 的交互项回归系数为正且显著，说明当政府税收优惠强度较高时，环境规制对企业创新绩效 有明显的正向促进作用。而且，政府税收优惠自身对创新绩为负向影响，但当其与环境规制 共同发挥作用时，可以显著促进企业创新绩效的提高。

\section{5. 结论与政策建议}

从上述 2011-2015 年我国制造业上市公司微观层面的面板数据进行全样本与分组的研究 结果可以得到如下结论及对应的政策建议:

环境规制与企业创新绩效之间的关系为倒“U”型，即适当的环境规制有利于企业创新，超 过临界点将不利于企业创新。因此，政府应该将环境规制政策及措施控制在合理的尺度。政 府直接资金补贴对企业创新绩效的影响显著为正，政府税收优惠的影响则为负且不显著。因 此政府应该对增加直接资金补贴强度。第在全样本情形下，政府税收优惠与环境规制的交互 作用显著为正，而政府直接资金补贴的交互作用不显著。说明短期轻微的环境规制不利于企 业创新，长期强有力的环境规制有利于企业创新，此时若增加政府税收优惠可以正向且显著 促进企业创新绩效的提高。政府可以通过调整产业结构, 整合行业资源, 促进要素的合理配 置，使效率高的资源向效率高的企业集聚，提高其创新能力。

\section{致谢}

本研究由国家社科基金资助（批准号：17BGL019）：制造企业政府创新支持，开放创新 
模式与创新绩效关系研究

\section{References}

[1] ARMANDO S V. An Empirical Analysis of the Nonlinear Relationship between Environment Regulation and Manufacturing Productivity [J]. Journal of Applied Economics, 2013, 16(2):357-372.

[2] Mohr R D. Technical Change, External Economies and the Porter Hypotheses [J]. Journal of Environmental Economics and Management, 2002, 43(1):158-168.

[3] Jia Ruiyue, Wei Yuchang, Zhao Dingtao. Environmental regulation and technological progress in production: An empirical analysis based on regulatory tools[J]. Journal of China University of Science and Technology, 2013, 43(3):217-222.

[4] Chintrakarn P. Environmental Regulation and US States' Technical in Efficiency [J]. Economics Letters, 2008, 100(3):363-365.

[5] Wang Yuzhen,Xu Zhen.Effect of environmental policy on corporate performance[J].China Environmental Management,2012(05):5-14.

[6] Guellec D, Pottelsberghe B V, Potterie D L. The Impact of Public R\&D Expenditure on Business R\&D [J]. Economic Innovation New Technology, 2003(3): 225-243.

[7] Zhang Tongbin, Gao Tiemei. Tax incentives, high-tech industry development, and industrial restructuring[J]. Economic Research,2012(5):58-70.

[8] Bloom N, Griffith N, Reenen J V. Do R\&D Tax Credits Work? Evidence from a Panel of Countries 1979-1997 [J]. Journal of Public Economics, 2002, 85(1):1-31.

[9] Yu Yongze. Innovation Factor Agglomeration, Government Support and Innovation Efficiency of Science and Technology--A Spatial Panel Analysis Based on Provincial Data[J]. Economic Review, 2011(2):93-101.

[10]Lanoie P, et al. Environmental Policy, Innovation and Performance: New Insights on the Porter Hypothesis [J]. Journal of Economics and Management Strategy, 2011, 20:803-42.

[11]Kneller R, Manderson E. Environmental Regulations and Innovation Activity in UK Manufacturing Industries [J]. Resource and Energy Economics, 2012, 34(2):211-235.

[12] Mateescu C, Constantinescu I, Alves M. Environmental Hazards and Anaerobic Treatment of Waste Waters Generated in Alcohol Industry [J]. Environmental Engineering and Management Journal, 2010, (9): 393-397.

[13] Shen Neng. Environmental Efficiency, Industry Heterogeneity and Optimal Regulation Intensity--China's Industrial Enterprise Panel Data Linearity Test [J]. China's Industrial Economy, 2012,3:56-68.

[14] Li Na, Wu Shidai. The impact of expanding openness and environmental regulation on the upgrading of China's industrial structure[J]. Economic Geography, 2016, 36(11): 109-123.

[15]Dundas N H, Roper S. Output Additionality of Public Support for Innovation: Evidence for Irish Manufacturing Plants [J]. European Planning Studies, 2010(1):107-122.

[16]Kleer R. Government R\&D Subsidies as a Signal for Private Investors [J]. Research Policy, 2010, 39:1361-1374.

[17]Guo Xiaodan, He Wenxuan, Xiao Xingzhi. Government subsidies, additional behaviors and changes in $\mathrm{R} \& \mathrm{D}$ activities in strategic emerging industries[J].Macroeconomic Studies, 2011(11):63-69+111.

[18]ZENG Ping, GENG Hong. Government Support and Enterprise Innovation: Research Review and Future Outlook[J]. Research and Development Management, 2014, 26(2):98-109.

[19]Murphy K M, Shleifer A, Vishny R W. Why is Rent Seeking so Costly to Growth? [J].The American Economic Review, 1993, 83(2):409-414.

[20]Fan Yunqi, Li Xiaozhong.Government R\&D Input, Space Spillover, and High-tech Industry 
Technological Innovation Efficiency in China[J].Industrial Technology Economics, 2014(5):101-107.

[21]Sun Wei,Jiang Sanliang.Research on Innovation Effect of Environmental Regulation and Government Investment[J].Economic Observation,2015,29(12):106-111. 